\title{
Topological field theories and their symmetries within the Batalin-Vilkovisky framework
}

\author{
F. Gieres, ${ }^{1}$ J. M. Grimstrup, ${ }^{2}$ H. Nieder ${ }^{3}$ T. Pisar, ${ }^{2}$ and M. Schweda ${ }^{2}$ \\ ${ }^{1}$ Institut de Physique Nucléaire, Université Claude Bernard, 43, boulevard du 11 novembre 1918, F-69622-Villeurbanne, France \\ ${ }^{2}$ Institut für Theoretische Physik, Technische Universität Wien, Wiedner Hauptstrasse 8-10, A-1040 Wien, Austria \\ ${ }^{3}$ Theory Division, CERN, CH-1211-Geneva 23, Switzerland
}

(Received 10 December 2001; revised manuscript received 3 May 2002; published 31 July 2002)

\begin{abstract}
We discuss the algebraic construction of topological models (of both Schwarz- and Witten-type) within the Batalin-Vilkovisky formalism and we elaborate on a simple description of vector supersymmetry within this framework.
\end{abstract}

DOI: 10.1103/PhysRevD.66.025027

PACS number(s): 11.10.Kk, 11.15.Bt, 11.10.Gh

\section{INTRODUCTION}

The present paper is devoted to the algebraic construction [1] and to the symmetries of topological field theories of Schwarz-type (see [2] for a review of the latter theories). The classical action of some of these models (e.g., $B F$ models in a space-time of dimension $d \geqslant 4[3,4]$ ) admits on-shell reducible symmetries and thus leads to a Becchi-Rouet-StoraTyutin (BRST) operator which is only nilpotent on shell. Moreover, the gauge-fixed action for these models admits a supersymmetry-like invariance, the so-called vector supersymmetry (VSUSY), which also generates an on-shell algebra [5-8]. Such on-shell invariances raise problems upon quantization of these theories.

The Lagrangian Batalin-Vilkovisky (BV) formalism represents a systematic approach to this problem [9]. (For a short summary, see Ref. [10].) In fact, in this canonical (symplectic) setting, all fields are supplemented from the beginning on with antifields and these additional variables ensure the off-shell closure of symmetry algebras. Thus, antifields somehow play the same role as auxiliary fields in supersymmetric field theories $[7,10]$. The antifields of the BV formalism correspond to the external sources of the standard BRST approach and can be expressed in terms of the latter. In this way, one recovers symmetry algebras for the basic fields whose closure is guaranteed by the external sources. The corresponding symmetry transformations coincide with those obtained in the standard BRST-approach by the action of the linearized Slavnov-Taylor operator.

As a matter of fact [2,7], topological field theories of Schwarz-type provide a neat application for the BV formalism which is often discussed in quite general terms in the literature [11]. The algebraic approach to this formalism was pioneered by Ikemori [1] and applied to various models in the sequel $[12,13]$ (see also [14] for earlier work and [15] for an interesting field-theoretic interpretation). We will incorporate VSUSY in this framework and show that this algebraic treatment of VSUSY yields a major simplification with respect to a similar approach within the BRST formalism [16], thus simplifying the study of the renormalization and finiteness properties of topological models [17].

In the present work, we restrict our attention to models in flat $d$-dimensional space-time, but a generalization of VSUSY to arbitrary manifolds can be achieved [18]. In fact, the latter allows us to tackle the relationship between $B F$ models and gravity $[3,19]$ where a VSUSY-like invariance also exists [20].

The fundamental ingredients of the algebraic approach $[1,12]$ are extended forms (corresponding to the complete ladders of the BV formalism): the latter can be used to write down action functionals as well as horizontality conditions or Russian formulas $[21,22]$ summarizing the basic symmetries of the action. The essential tool for describing VSUSY transformations is given by the so-called $\varnothing$-symmetry conditions introduced in Ref. [16].

Our paper is organized as follows. In Sec. II, we briefly recall the BV approach and the geometric framework which is generally chosen for its formulation. In Sec. III, we formulate general principles for the algebraic construction of topological models and summarize the different classes of topological models of both Schwarz- and Witten-type which can be obtained along these lines. Moreover, we will outline the derivation of VSUSY-transformations within the algebraic approach to the BV formalism. To anticipate our conclusions in this respect, we already indicate that this procedure allows us to recover various known results (or slight generalizations thereof) almost effortless, in a systematic way and in a quite compact form. As a by-product, we will also present a novel interpretation of the VSUSY-algebra in Sec. IV C. For concreteness, 3D Chern-Simons theory is occasionally considered for illustrative purposes and the 4D $B F$ model is discussed in detail in the Appendix: this also allows for a comparison with previous studies within the BRST or BV setting. (Since this topological model admits an on-shell reducible symmetry, it exhibits the typical features of such models.)

\section{BV APPROACH TO FIELD THEORIES WITH LOCAL SYMMETRIES}

\section{A. General setting}

The field theoretic models to be discussed admit a Lie algebra of symmetries and the involved fields are $p$ forms with values in this Lie algebra. In particular, we always have the Yang-Mills connection 1-form $A$ and the associated curvature 2 -form $F=d A+\frac{1}{2}[A, A]$. The field strength of a Lie algebra-valued field $\varphi$ other than $A$ is given by its covariant derivative $D \varphi=d \varphi+[A, \varphi]$. We will only be concerned with 
the classical theory and the fields occurring in the initial invariant action of a model will be referred to as classical fields.

\section{B. A simple example: Chern-Simons theory in $\mathbf{R}^{3}$}

One of the simplest examples of a topological field theory (of Schwarz-type) is given by the Chern-Simons theory in $\mathbf{R}^{3}$. The corresponding action

$$
S_{\text {inv }}[A]=\frac{1}{2} \int_{\mathbf{R}^{3}} \operatorname{tr}\left\{A d A+\frac{2}{3} A A A\right\}
$$

is invariant under infinitesimal gauge transformations, $\delta A$ $=D c$, and it leads to the equation of motion $F=0$, i.e., a zero-curvature condition for the connection $A$. In expression (1) and in the following, the wedge product sign is omitted.

The gauge invariance of the functional (1) represents an off-shell, irreducible symmetry and therefore the BV description of this invariance leads, up to minor modifications summarized in the Appendix, to the same results as the BRST approach.

\section{Geometric framework of BRST and BV approaches}

Let us briefly recall the conventions and notation that are generally used for the geometric framework of the standard BRST approach $[8,22]$. In this setting, infinitesimal symmetry parameters are turned into ghost fields. Thus, the geometric sector of the Chern-Simons model involves the classical field $A$ and the ghost field $c$ associated with infinitesimal gauge transformations. Lower and upper indices of a field label its form degree and ghost number, respectively. For each field, the ghost number is added to the form degree in order to define a total degree and all commutators are assumed to be graded with respect to this degree. The BRSToperator $s$ increases the ghost number by one unit, but it does not modify the form degree of fields. In view of the definition of Green functions or the formulation of the Slavnov-Taylor identity, one also introduces external sources $\gamma, \sigma, \ldots$ which couple to the (nonlinear) BRST transformations of the fields $A, c, \ldots$ belonging to the geometric sector: for the Chern-Simons theory, this simply amounts to the addition of the term

$$
S_{\text {ext }}=\int_{\mathbf{R}^{3}} \operatorname{tr}\left\{\gamma_{2}^{-1} s A+\sigma_{3}^{-2} s c\right\}
$$

to the action. The latter contribution is $s$ invariant since the operator $s$ is nilpotent and since external fields are assumed to be $s$ inert.

In the $B V$ approach to Chern-Simons theory, one starts with the basic fields $\left(\Phi^{a}\right)=(A, c)$ together with the corresponding antifields $\left(\Phi_{a}^{*}\right)=\left(A^{*}, c^{*}\right) \equiv\left(A_{2}^{-1}, A_{3}^{-2}\right)$ which have the same index structure as the sources $\left(\gamma_{2}^{-1}, \sigma_{3}^{-2}\right)$. All of these fields then define the geometric or minimal sector of the theory. Thereafter, $s$ variations are defined for all of these variables, the transformations of the antifields being non trivial. After carrying out the gauge-fixing procedure, all antifields $\left(A^{*}, c^{*}, \ldots\right)$ can be eliminated in terms of exter- nal sources $(\gamma, \sigma, \ldots)$ : this allows us to recover essentially the same results as those given by the Slavnov-Taylor identity of the BRST approach-see Appendix 1.5. for more details.

\section{BV algorithm}

The $B V$ algorithm starts with a classical action $S_{\text {inv }}[A, \ldots]$ which is defined on $d$-dimensional space-time $\mathcal{M}_{d}$ and which is invariant under some BRST transformations denoted by $s_{0} \Phi^{a}$. For many models, the operator $s_{0}$ is only nilpotent on the mass-shell. The goal then consists of determining a so-called minimal action

$$
S_{\min }\left[\Phi^{a} ; \Phi_{a}^{*}\right]=S_{\mathrm{inv}}[A, \ldots]+\sum_{q=1}^{n} \Phi_{a_{1}}^{*} \ldots \Phi_{a_{q}}^{*} \Delta_{q}\left[\Phi^{a}\right]
$$

satisfying the classical BV master equation

$$
\int_{\mathcal{M}_{d}} \operatorname{tr}\left\{\frac{\delta S_{\min }}{\delta \Phi^{a}} \frac{\delta S_{\min }}{\delta \Phi_{a}^{*}}\right\}=0
$$

and generating nilpotent $s$ variations by virtue of the definitions

$$
s \Phi^{a}=-\frac{\delta S_{\min }}{\delta \Phi_{a}^{*}}, \quad s \Phi_{a}^{*}=-\frac{\delta S_{\min }}{\delta \Phi^{a}} .
$$

Thus, the minimal action represents an s-invariant action extending the classical action,

$$
S_{\mathrm{inv}}[A, \ldots] \equiv S_{\min }\left[\Phi^{a}, \Phi_{a}^{*}=0\right],
$$

and the $s$ variations extend the standard BRST transformations in the sense that $s_{0} \Phi^{a}=\left.\left(s \Phi^{a}\right)\right|_{\Phi_{a}^{*}=0}$. (Accordingly, the classical equations of motion can be viewed as a consequence of the choice $\Phi_{a}^{*}=0=s \Phi_{a}^{*}$.)

\section{E. Gauge-fixing procedure}

The gauge degrees of freedom of the models have to be fixed by virtue of some gauge-fixing conditions $\mathcal{F}_{\alpha}=0$. The latter are implemented by introducing a gauge-fermion $\Psi_{\mathrm{gf}}$ of ghost number -1 depending on antighost fields $\bar{C}^{\alpha}$ :

$$
\Psi_{\mathrm{gf}}=\int_{\mathcal{M}_{d}} \operatorname{tr}\left\{\bar{C}^{\alpha} \mathcal{F}_{\alpha}\right\}
$$

The $s$ variation of $\bar{C}^{\alpha}$ yields the multiplier field $\Pi^{\alpha}$,

$$
s \bar{C}^{\alpha}=\Pi^{\alpha}, \quad s \Pi^{\alpha}=0,
$$

and the corresponding antifields are assumed to transform "the other way round:"

$$
s \Pi_{\alpha}^{*}=(-1)^{(d+1)\left(\left|\bar{C}^{\alpha}\right|+1\right)} \bar{C}_{\alpha}^{*}, \quad s \bar{C}_{\alpha}^{*}=0 .
$$

(Here and in the following, the total degree of a field $\varphi$ is denoted by $|\varphi|$.) These trivial ("contractible") BRST dou- 
blets, which do not contribute to the physical content of the theory, are taken into account by adding an auxiliary fields contribution

$$
S_{\text {aux }}\left[\bar{C}_{\alpha}^{*}, \Pi^{\alpha}\right]=-\int_{\mathcal{M}_{d}} \operatorname{tr}\left\{\bar{C}_{\alpha}^{*} \Pi^{\alpha}\right\}
$$

to the action $S_{\min }$. The resulting nonminimal action depends on the fields $\left(\Phi^{A}\right)=\left(\Phi^{a}, \bar{C}^{\alpha}, \Pi^{\alpha}\right)$ and the corresponding antifields $\left(\Phi_{A}^{*}\right)=\left(\Phi_{a}^{*}, \bar{C}_{\alpha}^{*}, \Pi_{\alpha}^{*}\right)$ :

$$
S_{\mathrm{nm}}\left[\Phi^{A}, \Phi_{A}^{*}\right]=S_{\min }\left[\Phi^{a}, \Phi_{a}^{*}\right]+S_{\mathrm{aux}}\left[\bar{C}_{\alpha}^{*}, \Pi^{\alpha}\right] .
$$

The master equation (2) and the $s$ variations (3) still hold if $S_{\min }$ is extended to $S_{\mathrm{nm}}$ and $\Phi^{a}$ to $\Phi^{A}$.

The elimination of all antifields $\left(\Phi_{A}^{*}\right)$ in terms of external sources $\left(\rho_{A}\right)$ is performed according to the general prescription

$$
\Phi_{A}^{*}=-\hat{\rho}_{A} \equiv-\rho_{A}+(-1)^{(d+1)\left|\Phi^{A}\right|+d} \frac{\delta \Psi_{\mathrm{gf}}}{\delta \Phi^{A}} .
$$

After substituting these expressions into the nonminimal BV action (9), one obtains the vertex functional:

$$
\Sigma\left[\Phi^{A}, \rho_{A}\right]=\left.S_{\mathrm{nm}}\right|_{A} ^{*}=S_{\mathrm{inv}}+S_{\mathrm{gf}}+S_{\mathrm{ext}}+S_{\text {quadr }} .
$$

Here, the term $S_{\mathrm{gf}}=s \Psi_{\mathrm{gf}}$ denotes the gauge-fixing part for the classical, gauge invariant action $S_{\text {inv }}$, the contribution $S_{\text {ext }}$ represents the linear coupling of the external sources $\rho_{A}$ to the $s$ variations of the fields $\Phi^{A}$, and $S_{\text {quadr }}$ is an additional term (that is nonlinear in the sources) which appears in the case of on-shell reducible symmetries.

\section{F. BV brackets and master equation}

As in Eq. (9), let $\left(\Phi^{A}\right)=\left(\Phi^{a}, \bar{C}^{\alpha}, \Pi^{\alpha}\right)$ collectively denote all fields, i.e., the classical and ghost fields $\left(\Phi^{a}\right)$ defining the minimal sector, the antighosts $\bar{C}^{\alpha}$, and the multiplier fields $\Pi^{\alpha}$. Accordingly, let $\left(\Phi_{A}^{*}\right)=\left(\Phi_{a}^{*}, \bar{C}_{\alpha}^{*}, \Pi_{\alpha}^{*}\right)$ denote the associated antifields and let $\left(\Theta^{A}\right)=\left(\Phi^{A} ; \Phi_{A}^{*}\right)$. Quite generally, if $\Phi^{A}$ has index structure $\left(\Phi^{A}\right)_{p}^{g}$, then the corresponding antifield has index structure $\left(\Phi_{A}^{*}\right)_{d-p}^{-g-1}$ where $d$ denotes the space-time dimension. Accordingly, for a space-time $\mathcal{M}_{d}$ of odd (even) dimension, the fields and their antifields have the same (opposite) parity.

For any two functionals $X\left[\Theta^{A}\right]$ and $Y\left[\Theta^{A}\right]$ of the fields $\left(\Theta^{A}\right)$, the $B V$ bracket is the graded bracket defined by

$$
\{X, Y\}=\int_{\mathcal{M}_{d}} \operatorname{tr}\left\{\frac{\delta X}{\delta \Phi^{A}} \frac{\delta Y}{\delta \Phi_{A}^{*}} \pm \frac{\delta Y}{\delta \Phi^{A}} \frac{\delta X}{\delta \Phi_{A}^{*}}\right\},
$$

where the sign depends on the Grassmann parity of $X$ and $Y$. (Our convention to use left functional derivatives differs from the one which is frequently used in the literature [11].)

Let $\Gamma\left[\Phi^{A}, \Phi_{A}^{*}\right]$ be the nonminimal BV action (9) as defined on $\mathcal{M}_{d}$. As noted above, the latter is a solution of the classical BV master equation

$$
\{\Gamma, \Gamma\}=0, \quad \text { i.e., } \int_{\mathcal{M}_{d}} \operatorname{tr}\left\{\frac{\delta \Gamma}{\delta \Phi^{A}} \frac{\delta \Gamma}{\delta \Phi_{A}^{*}}\right\}=0,
$$

and the $s$ variations of fields and antifields are given by

$$
\begin{gathered}
s \Theta^{A}=\left\{\Gamma, \Theta^{A}\right\}, \quad \text { i.e., } s \Phi^{A}=-\frac{\delta \Gamma}{\delta \Phi_{A}^{*}} \\
\text { and } s \Phi_{A}^{*}=-\frac{\delta \Gamma}{\delta \Phi^{A}} .
\end{gathered}
$$

By virtue of $s \Gamma=\{\Gamma, \Gamma\}$, the master equation (13) simply expresses the $s$ invariance of $\Gamma$. The off-shell nilpotency of the $s$ operator can be viewed as a consequence of the graded Jacobi identity for the BV bracket.

Since the implementation of the gauge-fixing conditions follows a canonical procedure [see Eqs. (5)-(11)], the nontrivial part of the problem consists of determining the functional $S_{\min }$, i.e., a solution of the master equation which only depends on the variables $\left(\Phi^{a} ; \Phi_{a}^{*}\right)$. The next section presents an algebraic procedure for constructing this functional for topological models.

\section{ALGEBRAIC APPROACH TO TOPOLOGICAL MODELS AND TO THEIR SYMMETRIES}

After presenting some general principles summarizing the algebraic construction of topological models, we will investigate which models can be constructed using this approach and determine their characteristic features.

\section{A. Geometric framework}

To start with, we recall the general framework for describing a gauge field and, more generally, $p$-form potentials, on $d$-dimensional space-time $\mathcal{M}_{d}[1,12]$.

\section{Generalized fields and duality}

In the BRST approach, the ghost field $c$ is added to the connection $A$ in order to define the generalized Yang-Mills field $\widetilde{A}=A+c$. In the BV approach, one includes fields of all form degrees which are allowed by the dimension of spacetime [1] so as to obtain the generalized field (or extended form or complete ladder)

$$
\widetilde{A}=A_{d}^{1-d}+A_{d-1}^{2-d}+\cdots+A_{2}^{-1}+A+c,
$$

e.g., in three dimensions:

$$
\widetilde{A}=A_{3}^{-2}+A_{2}^{-1}+A+c=c^{*}+A^{*}+A+c .
$$

Thus, generalized fields also involve fields with negative ghost number (which are to be interpreted as antifields).

More specifically, the 3D Yang-Mills ladder (16) can be viewed as a "self-dual" quantity since it involves the basic fields $A$ and $c$ together with their antifields [1,12]. To make this notion of duality more precise [12], we consider a 
$p$-form gauge potential $X_{p} \equiv X_{p}^{0}$ with $p \in\{0,1, \ldots, d\}$. This field gives rise to a generalized form

$$
\begin{aligned}
\widetilde{X}_{p}= & \sum_{q=0}^{d} X_{d-q}^{p-d+q}=X_{d}^{p-d}+X_{d-1}^{p-d+1}+\cdots+X_{p} \\
& +\cdots+X_{0}^{p},
\end{aligned}
$$

which involves all ghosts and "ghosts for ghosts," as well as some fields with negative ghost number. However, in general, the index structure of the latter fields does not allow us to identify them with the antifields associated to the fields appearing in $\widetilde{X}_{p}$. Rather one has to introduce a so-called dual form $\widetilde{Y}_{d-p-1}$ with an analogous expansion,

$$
\begin{aligned}
\widetilde{Y}_{d-p-1}= & \sum_{q=0}^{d} Y_{d-q}^{-p-1+q}=Y_{d}^{-p-1}+\cdots+Y_{d-p-1} \\
& +\cdots+Y_{0}^{d-p-1} .
\end{aligned}
$$

The generalized forms (17) and (18) are dual to each other in the sense that the fields with negative ghost number contained in the first one are the antifields associated to the fields with positive ghost number contained in the second one and vice versa: i.e.,

$$
\begin{gathered}
\left(X_{p-q}^{q}\right) *=Y_{d-(p-q)}^{-q-1} \text { for } q=0, \ldots, p, \\
\left(Y_{d-p-1-q}^{q}\right)^{*}=X_{p+1+q}^{-q-1} \text { for } q=0, \ldots, d-p-1 .
\end{gathered}
$$

For instance, for $d=3$, the Yang-Mills ladder $\widetilde{A} \equiv \widetilde{X}_{1}$ is selfdual and, for $d=4$, this ladder is dual to the 2-form potential ladder $\widetilde{B} \equiv \widetilde{Y}_{2}$ (the potentials $A$ and $B$ being the basic variables of the 4D $B F$ model, see Appendix).

\section{Generalized derivatives and field strengths}

The $s$ differential is added to the exterior derivative $d$ so as to define the generalized derivative $\widetilde{d}=d+s$. In the same vein, one introduces the generalized field strengths of $\widetilde{A}$ and $\varphi$ :

$$
\widetilde{F}=\widetilde{d} \widetilde{A}+\frac{1}{2}[\widetilde{A}, \widetilde{A}], \quad \widetilde{D} \varphi=\widetilde{d} \varphi+[\widetilde{A}, \varphi] .
$$

Actually, it also proves useful [12] to define the generalized fields

$$
F^{\tilde{A}}=d \widetilde{A}+\frac{1}{2}[\widetilde{A}, \widetilde{A}], \quad D^{\widetilde{A}} \varphi=d \varphi+[\widetilde{A}, \varphi]
$$

which satisfy the Bianchi identities

$$
D^{\tilde{A}} F^{\tilde{A}}=0, \quad D^{\tilde{A}} D^{\tilde{A}} \varphi=\left[F^{\tilde{A}}, \varphi\right] .
$$

\section{B. Field content and construction of models}

All of the models to be considered involve a gauge field $A$, hence a generalized form $\widetilde{A} \equiv \widetilde{X}_{1}$ and the dual form $\widetilde{B}$ $\equiv \widetilde{Y}_{d-2}$. Some models contain additional dual pairs $\left(\widetilde{X}_{p}, \widetilde{Y}_{d-p-1}\right)$ with $0 \leqslant p \leqslant d-1$ which are coupled to the generalized gauge field $\widetilde{A}$.

We now summarize the general algebraic procedure for constructing models $[1,12]$.

(1) One imposes horizontality conditions on $\widetilde{A}, \widetilde{B}, \widetilde{X}, \widetilde{Y}$, i.e., conditions on their field strengths $\widetilde{F}, \widetilde{D} \widetilde{B}, \widetilde{D} \widetilde{X}, \widetilde{D} \widetilde{Y}$ which are compatible with the Bianchi identities

$$
\widetilde{D} \widetilde{F}=0 \quad \text { and } \quad \widetilde{D}^{2} \widetilde{\Omega}=[\widetilde{F}, \widetilde{\Omega}] \text { for } \quad \widetilde{\Omega}=\widetilde{B}, \widetilde{X}, \widetilde{Y} .
$$

This determines nilpotent $s$ variations for the components of $\widetilde{A}, \widetilde{B}, \widetilde{X}, \widetilde{Y}$. (For instance, for the Chern-Simons theory, the horizontality condition reads $\widetilde{F}=0$ and is equivalent to $s \widetilde{A}$ $=-F^{\tilde{A}}$.) In practice, the horizontality conditions are nothing but the tilted equations of motion of the model to be defined in the next step: thus, the horizontality conditions fix both the symmetries and the dynamics.

(2) One looks for a generalized Lagrangian density $\widetilde{\mathcal{L}}$, i.e., a generalized $p$ form which depends polynomially on $\widetilde{A}, \widetilde{B}, \ldots$ and their exterior derivatives $d \widetilde{A}, d \widetilde{B}, \ldots$ and which satisfies the cocycle condition $s \widetilde{\mathcal{L}}=d(.$.$) where s$ denotes the operator defined in the first step. By integrating the $d$-form part of $\widetilde{\mathcal{L}}$ over space-time, we obtain the s-invariant action

$$
\left.S_{\min }\left[\Phi^{a}, \Phi_{a}^{*}\right] \equiv \int_{\mathcal{M}_{d}} \widetilde{\mathcal{L}}\right|_{d} ^{0},
$$

from which we can infer the classical action by virtue of the projection (4). The $s$ variations defined in the first step coincide with those generated by the functional (23) according to relations (3), i.e., $S_{\min }$ solves the BV master equation. (For instance, for the Chern-Simons theory on $\mathbf{R}^{3}$, the cocycle condition

$$
s \operatorname{tr}\left\{\widetilde{A} d \widetilde{A}+\frac{2}{3} \widetilde{A}^{3}\right\}=-d \operatorname{tr}\left\{\widetilde{A} d \widetilde{A}+\frac{1}{3} \widetilde{A}^{3}\right\}
$$

yields the minimal BV action

$$
\begin{aligned}
S_{\min }\left[\Phi^{a} ; \Phi_{a}^{*}\right] & \left.\equiv \frac{1}{2} \int_{\mathbf{R}^{3}} \operatorname{tr}\left\{\widetilde{A} d \widetilde{A}+\frac{2}{3} \widetilde{A} \widetilde{A} \widetilde{A}\right\}\right|_{3} ^{0} \\
& \left.=S_{\text {inv }}[A]-\int_{\mathbf{R}^{3}} \operatorname{tr}\left\{\Phi_{a}^{*} s \Phi^{a}\right\} .\right)
\end{aligned}
$$

Since the $s$ operator is defined in terms of conditions involving the covariant quantities $\widetilde{F}, \widetilde{D} \widetilde{B}, \ldots$, the polynomial $\widetilde{\mathcal{L}}$ depends on $d \widetilde{A}, d \widetilde{B}, \ldots$ by virtue of the field strengths $F^{\tilde{A}}, D^{\widetilde{A}} \widetilde{B}, \ldots$. By construction, the classical action (4) is invariant under the standard BRST transformations $s_{0} \Phi^{a}$ $\left.\equiv\left(s \Phi^{a}\right)\right|_{\Phi_{a}^{*}=0}$. If $s \Phi^{a}$ involves $\Phi_{a}^{*}$, then $s_{0}$ is only nilpotent on the mass shell. 
Obviously, the algebraic approach proceeds in the opposite order than the usual BV-algorithm summarized above [12]. It raises some general questions to which we will come back to at the end of Sec. III.

\section{Examples}

Mostly following references [1,12], we will now present an overview of models which can be constructed by the procedure outlined above. As pointed out by Baulieu $[12,13]$, this construction not only yields topological field theories of Schwarz-type, but also theories of Witten-type. We will not spell out the explicit form of the cocycle condition for each model, since the latter can easily be obtained from the given Lagrangian $\widetilde{\mathcal{L}}$ by application of the $s$ operator. However, we note that (contrary to the indications in [12]), the cocycle condition does not always have the simple form $(s+d) \widetilde{\mathcal{L}}$ $=0$ as illustrated by Eq. (24). The compatibility of horizontality conditions can readily be verified for each example.

\section{BF model in $d \geqslant 2$}

This model involves the pair $(\widetilde{A}, \widetilde{B})$, the action being given by

$$
S_{\text {min }}=\left.\int_{\mathcal{M}_{d}} \operatorname{tr}\left\{\widetilde{B} F^{\widetilde{A}}\right\}\right|_{d} ^{0}=\int_{\mathcal{M}_{d}} \operatorname{tr}\{B F\}+\text { s.t., }
$$

where s.t. stands for source (or rather antifield) terms. The classical equations of motion are the zero-curvature conditions $F=0=D B$. The 4D model is worked out in detail in the Appendix. For $d=2$, the field $B$ represents a 0 -form and does not have a local gauge symmetry (apart from ordinary gauge transformations) $[2,3,7]$.

\section{BF model with cosmological constant}

For $d=3$ and $d=4$, a term involving a real dimensionless parameter $\lambda$ can be added [3] to the $B F$ action for the pair $(\widetilde{A}, \widetilde{B})$. For $\mathbf{d}=\mathbf{3}$, the minimal action reads [1]

$$
\begin{aligned}
S_{\min } & =\left.\int_{\mathcal{M}_{3}} \operatorname{tr}\left\{\widetilde{B} F^{\tilde{A}}+\frac{\lambda}{3} \widetilde{B}^{3}\right\}\right|_{3} ^{0} \\
& =\int_{\mathcal{M}_{3}} \operatorname{tr}\left\{B F+\frac{\lambda}{3} B^{3}\right\}+\text { s.t., }
\end{aligned}
$$

which leads to the classical equations of motion $F+\lambda B^{2}$ $=0=D B$.

For $\mathbf{d}=\mathbf{4}$, the action

$$
\begin{aligned}
S_{\text {min }} & =\left.\int_{\mathcal{M}_{4}} \operatorname{tr}\left\{\widetilde{B} F^{\tilde{A}}+\frac{\lambda}{2} \widetilde{B}^{2}\right\}\right|_{4} ^{0} \\
& =\int_{\mathcal{M}_{4}} \operatorname{tr}\left\{B F+\frac{\lambda}{2} B^{2}\right\}+\text { s.t. }
\end{aligned}
$$

leads to the complete equations of motion $F^{\widetilde{A}}+\lambda \widetilde{B}=0$ $=\widetilde{D} \widetilde{B}$. From these, we can deduce, amongst others, that

$$
s B^{*}=-\left[c, B^{*}\right]-(F+\lambda B) .
$$

We now impose the complete equation of motion for the 2 -form potential $B$, i.e.,

$$
0=\left.\left(F^{\widetilde{A}}+\lambda \widetilde{B}\right)\right|_{2} ^{0}=\frac{\delta S_{\min }}{\delta B}=-s B^{*} .
$$

This implies that the field $B^{*}$ can be set to zero consistently. In addition, we choose $\lambda=-1$ for the sake of simplicity. From Eqs. (30) and (29), we then conclude that $B=F$ and, by substitution into Eq. (28), we obtain

$$
\begin{aligned}
S_{\min } \mid \delta S_{\min } / \delta B=0 & =\frac{1}{2} \int_{\mathcal{M}_{4}} \operatorname{tr}\{F F\} \\
& +\sum_{\Phi^{a}=A, c, B_{1}, B_{0}} \int_{\mathcal{M}_{4}} \operatorname{tr}\left\{\Phi_{a}^{*} s \Phi^{a}\right\} .
\end{aligned}
$$

This expression represents the minimal action associated with the topological invariant $\int_{\mathcal{M}_{4}} \operatorname{tr}\{F F\}$ whose gauge fixing gives rise to Witten's topological Yang-Mills (TYM) theory [2]. For the latter theory, both the BRST algebra [23] and the VSUSY algebra [16,24] close off-shell for different classes of gauge-fixings so that the introduction of antifields does not seem useful for studying the quantization of this model. Yet, it is quite interesting that the TYM theory whose gauge-fixing procedure refers to self-duality conditions can be obtained from an action involving only a dual pair of potentials [13].

\section{3a. BF-XY model}

For $d \geqslant 2$, one can add to the $B F$ model (26) some dual pairs $\left(\widetilde{X}_{p}, \widetilde{Y}_{d-p-1}\right)$ with $0 \leqslant p \leqslant d-1$ coupling to $\widetilde{A}$ according to $[12]$

$$
\begin{aligned}
S_{\min } & =\left.\int_{\mathcal{M}_{d}} \operatorname{tr}\left\{\widetilde{B} F^{\widetilde{A}}+\sum_{p=0}^{d-1} \widetilde{X}_{p} D^{\tilde{A}} \widetilde{Y}_{d-p-1}\right\}\right|_{d} ^{0} \\
& =\int_{\mathcal{M}_{d}} \operatorname{tr}\left\{B F+\sum_{p=0}^{d-1} X_{p} D Y_{d-p-1}\right\}+\text { s.t. }
\end{aligned}
$$

This action leads to the classical equations of motion $0=F$ $=D B-\sum_{p=0}^{d-1}(-1)^{p}\left[X_{p}, Y_{d-p-1}\right]=D X_{p}=D Y_{d-p-1}$. It represents a first order action that is analogous to threedimensional Chern-Simons theory.

\section{3b. BF-XY model with BX coupling}

For any $d \geqslant 2$, the 2-form potential $X_{2}$ appearing in the previous model can be coupled directly to $B$ [12] with strength $\alpha \in \mathbf{R}$ :

$$
\begin{aligned}
S_{\min } & =\left.\int_{\mathcal{M}_{d}} \operatorname{tr}\left\{\widetilde{B}\left(F^{\widetilde{A}}+\alpha \widetilde{X}_{2}\right)+\widetilde{X}_{2} D^{\widetilde{A}} \widetilde{Y}_{d-3}\right\}\right|_{d} ^{0} \\
& =\int_{\mathcal{M}_{d}} \operatorname{tr}\left\{B\left(F+\alpha X_{2}\right)+X_{2} D Y_{d-3}\right\}+\text { s.t. }
\end{aligned}
$$


The classical equations of motion then take the form $0=F$ $+\alpha X=D Y+\alpha B=D B-[X, Y]$. By elimination of $X$ from the action functional by virtue of its algebraic equation of motion, one gets a classical action of the form $\int_{\mathcal{M}_{d}} d \operatorname{tr}\left\{F Y_{d-3}\right\}$ which is analogous to TYM theory [12]. More specifically, for $d=3$, this action, $\int_{\mathcal{M}_{3}} d \operatorname{tr}\left\{F Y_{0}\right\}$ $=\int_{\mathcal{M}_{3}} \operatorname{tr}\left\{F D Y_{0}\right\}$ describes magnetic monopoles and its gauge-fixing via Bogomol'nyi's equations yields a topological model that is closely related to four-dimensional TYM theory [25].

For $\mathbf{d}=\mathbf{4}$, a "dual" form of the model (33) is obtained by exchanging the generalized fields $\widetilde{B}_{2}$ and $\widetilde{X}_{2}$ in the pairs $\left(\widetilde{A}_{1}, \widetilde{B}_{2}\right),\left(\widetilde{X}_{2}, \widetilde{Y}_{1}\right)$ :

$$
S_{\min }=\left.\int_{\mathcal{M}_{4}} \operatorname{tr}\left\{\widetilde{X}_{2}\left(F^{\tilde{A}}+\alpha \widetilde{B}_{2}\right)+\widetilde{B}_{2} D^{\tilde{A}} \widetilde{Y}_{1}\right\}\right|_{4} ^{0} .
$$

To this functional one can add a contribution $\left.\int \operatorname{tr} \mathcal{F}\left(\widetilde{B}_{2}\right)\right|_{4} ^{0}$ of the form $s \int \Delta_{4}^{-1}+\int d(\cdots)$. Different gauge fixings then allow us to recover the Lagrangian $\operatorname{tr}\{\tau F F\}$ for the TYM theory and the one of the dual theory defined by the duality transformation $\tau \rightarrow 1 / \tau$ (see Ref. [26] for this and the following points). The $\theta$ parameter of the theory can be adjusted by adding the topological invariant

$$
\begin{aligned}
S_{\text {top }} \equiv & \int_{\mathcal{M}_{4}} d \operatorname{tr}\left\{a\left(A d A+\frac{2}{3} A A A\right)+b F Y_{1}+c Y_{1} D Y_{1}\right\} \\
= & a \int_{\mathcal{M}_{4}} \operatorname{tr}\{F F\}+b \int_{\mathcal{M}_{4}} \operatorname{tr}\left\{F D Y_{1}\right\} \\
& +c \int_{\mathcal{M}_{4}} \operatorname{tr}\left\{D Y_{1} D Y_{1}+F\left[Y_{1}, Y_{1}\right]\right\}
\end{aligned}
$$

with appropriately chosen complex parameters $a, b, c$. The different formulations of the TYM theory in two and eight dimensions can be approached along the same lines.

\section{3c. BF-XY model with mixed couplings}

Several sets of pairs $\left(\widetilde{X}_{p}, \widetilde{Y}_{d-p-1}\right),\left(\widetilde{U}_{p}, \widetilde{V}_{d-p-1}\right), \ldots$ with $0 \leqslant p \leqslant d-1$ can be considered and coupled by terms of the form $[X, Y],[X, U], B[X, U], \ldots$ [13]. For concreteness, we consider $d=6$ and independent pairs $\left(\widetilde{A}, \widetilde{B}_{4}\right),\left(\widetilde{X}_{2}, \widetilde{Y}_{3}\right),\left(\widetilde{U}_{2}, \widetilde{V}_{3}\right),\left(\widetilde{U}_{2}^{c}, \widetilde{V}_{3}^{c}\right)$ with an action $S_{\min }$ $=S_{\text {inv }}+$ s.t. where

$$
\begin{aligned}
S_{\mathrm{inv}}= & \int_{\mathcal{M}_{6}} \operatorname{tr}\left\{B(F+X)+X\left(D Y+\left[U, U^{c}\right]\right)\right. \\
& \left.+U D V-U^{c} D V^{c}+V V^{c}\right\} .
\end{aligned}
$$

By substituting the algebraic equations of motion $0=F+X$ $=V+D U^{c}=V^{c}+D U$ into the latter functional, we obtain the following six-dimensional topological model of Wittentype [13]:

$$
\begin{aligned}
S_{\mathrm{inv}} & =\int_{\mathcal{M}_{6}} \operatorname{tr}\left\{D U D U^{c}-F\left[U, U^{c}\right]\right\}-\int_{\mathcal{M}_{6}} \operatorname{tr}\{F D Y\} \\
& =\int_{\mathcal{M}_{6}} d \operatorname{tr}\left\{U D U^{c}\right\}-\int_{\mathcal{M}_{6}} d \operatorname{tr}\{F Y\} .
\end{aligned}
$$

The first part of this action admits BRST and VSUSY algebras which close off shell and which have been studied in Refs. [13,27].

\section{3D Chern-Simons theory and extensions thereof}

For $d=3$, we can choose $\widetilde{X}_{1}=\widetilde{A}=\widetilde{Y}_{1}$ and consider the Chern-Simons theory, see Eq. (25). One can also combine this theory with the models considered above [12] or include a term $\int_{\mathcal{M}_{3}} \operatorname{tr}\left\{X_{1} D X_{1}\right\}$ [4]. The generalization of ChernSimons theory to an arbitrary dimension [4] may be discussed as well using the algebraic approach [28].

\section{Supersymmetric extensions of the previous models}

The algebraic formalism admits a supersymmetric extension [29] which should allow us to discuss the supersymmetric versions of the previous models, e.g., super $B F$ models (see [30] and references therein).

\section{General features and generalizations}

\section{General features}

One may wonder what kind of field theoretic models can be constructed by the algebraic procedure summarized above and which generic features are shared by all of the models that we listed. Obviously, their field content is given by p-form potentials and they involve at least the connection 1 -form $A$. Only in three dimensions, the corresponding extended form $\widetilde{A}$ contains solely $A$, its ghost $c$, and the associated antifields $A^{*}, c^{*}$. Thus, 3 -manifolds are the only ones for which a model involving solely a Yang-Mills potential can be constructed. (Actually, such models can be obtained indirectly in other dimensions by eliminating some fields, as illustrated in Sec. III C 2 above.)

Otherwise, a common feature of all models constructed above is that their minimal action (involving the classical fields $A, \ldots$, the ghosts $c, \ldots$, and the associated antifields $A^{*}, c^{*}, \ldots$ ) can be written directly in terms of generalized fields $\widetilde{A}, \ldots$ obeying some generalized zero-curvature conditions $0=\widetilde{F}=\cdots[31]$. This fact is related to the following one. The dynamics of fields is described by a metricindependent, first order action, the kinetic term being of the form $A d A, B d A, X d Y$ and the gauge invariant interaction being given by some polynomial of the fields. (General arguments supporting that this is the only class of examples have been put forward in Ref. [28].) All of these theories are of topological nature.

If some of the classical equations of motion are algebraic (and linear in the basic fields), as it is the case in Secs. III C 2, III C 3b, and III C3c then they imply all other equations of motion by application of the covariant derivative. Moreover, elimination of fields by some algebraic equations 
of motion then reduces the first order actions to second order actions analogous to the TYM theory, i.e., topological models of Witten-type.

\section{Other models and possible generalizations}

Some examples related to two-dimensional gravity have been studied in Refs. [12,31]. In this case, the components of the space-time metric are viewed as gauge potentials associated to the invariance under general coordinate transformations.

A further extension of the formalism is the following. Consider the case of an Abelian gauge group. The pairs of potentials $\left(\widetilde{X}_{p}, \widetilde{Y}_{d-p-1}\right)$ can be generalized to mixed dual pairs $\left(\widetilde{X}_{p}, \widetilde{d} \widetilde{Y}_{d-p-2}\right)$ and $\left(\widetilde{d} \widetilde{X}_{p}, \widetilde{Y}_{d-p-2}\right)$ each of which involves an Abelian potential and an Abelian field strength. Such pairs appear in the transgression construction of $(d$ +1 )-dimensional topological field theories from $d$-dimensional topological models [32].

A different generalization of the algebraic formalism consists of introducing incomplete ladders and deformations of the operator $\widetilde{d} \equiv d+s$ [33]. This approach allows us to discuss cohomological aspects of Yang-Mills-type theories or supersymmetric extensions thereof $[29,33]$.

Finally, we note that Yang-Mills theories can be formulated in terms of a first order action by deforming a $B F$ model [34]. Thus, the algebraic formalism discussed here should also be useful for describing these (nontopological) field theories.

\section{Some open questions}

The numerous examples presented above illustrate the pertinence of the principles summarized in Sec. III B. An intriguing question is why the whole procedure works and, more specifically, why the $s$ variations defined in the first step [i.e., by virtue of horizontality conditions] coincide with those encountered in the second step [i.e., those generated by the functional (23)]. A further, related question is whether the constructed solution of the master equation always represents a proper solution, i.e., one involving all of the local symmetries and of the associated reducibility identities. This question can be answered in the affirmative for the examples considered. This fact certainly relies on the principle that one considers complete ladders (or consistent truncations thereof) and horizontality conditions which are compatible with the corresponding Bianchi identities. A detailed answer to these questions seems to require a deeper geometric understanding of the BV framework in general [35], and of the algebraic construction of topological models in particular. Presumably the field theoretic formulation of Refs. [15,29] provides the appropriate setting for this endeavor.

\section{VSUSY}

For Chern-Simons theory, we can fix the gauge by imposing the Lorentz condition using a Landau-type gauge, i.e., by choosing the gauge-fixing condition $\mathcal{F}_{\alpha} \equiv d \star A=0$ (where $\star A$ denotes the Hodge-dual of the 1 -form $A$ ). This constraint is implemented using a trivial BRST doublet $\left(\bar{C}^{\alpha}, \Pi^{\alpha}\right)$ $\equiv(\bar{c}, b)$. The resulting gauge-fixed action is invariant under VSUSY transformations [5] and this symmetry is usually described by a Ward identity [8]. An invariance of the same type can be found in other topological models of Schwarztype $[7,8]$ or of Witten-type [16,24].

At the infinitesimal level, VSUSY transformations are described on $\mathbf{R}^{d}$ by an operator $\delta_{\tau}$ where $\tau \equiv \tau^{\mu} \partial_{\mu}$ represents a constant, $s$-invariant vector field of ghost-number zero. The variation $\delta_{\tau}$ acts as an antiderivation which lowers the ghost number by one unit and which anticommutes with $d$. The operators $s$ and $\delta_{\tau}$ satisfy a graded algebra of Wess-Zumino type:

$$
\left[s, \delta_{\tau}\right]=\mathcal{L}_{\tau}+\text { equations of motion. }
$$

Here, $\mathcal{L}_{\tau}=\left[i_{\tau}, d\right]$ denotes the Lie derivative with respect to the vector field $\tau$ and $i_{\tau}$ the interior product with $\tau$.

A general procedure for obtaining the explicit form of VSUSY transformations for topological models of Schwarztype is known $[8,36]$, but it is rather involved for complex models. Alternatively, the VSUSY variations can be derived within the standard BRST approach from the so-called $\varnothing$-type symmetry condition [16]

$$
\delta_{\tau} \widetilde{A}=i_{\tau} \tilde{A}
$$

(and $\delta_{\tau} \widetilde{B}=i_{\tau} \widetilde{B}$ for $p$-form potentials $B$ ). Though this approach works quite well for topological models of Wittentype [16], it is also involved for topological models of Schwarz-type due to the fact that it refers to the equations of motion. To avoid the latter complication, we will now apply this simple algebraic approach within the BV formalism in order to obtain VSUSY transformations as well as the induced variation of the $\mathrm{BV}$ action.

\section{A. Algebraic approach}

In the $B V$ approach, we can start from the symmetry condition (38), the only difference being that $\widetilde{A}$ now involves both fields of positive and negative ghost number. In the $3 \mathrm{D}$ case, substitution of the expansion (16) into Eq. (38) yields the VSUSY variations in the geometric sector,

$$
\delta_{\tau} c=i_{\tau} A, \quad \delta_{\tau} A=i_{\tau} A^{*}, \quad \delta_{\tau} A^{*}=i_{\tau} c^{*}, \quad \delta_{\tau} c^{*}=0 .
$$

We note that, if two fields are related by VSUSY (e.g., $c \stackrel{\delta_{\tau}}{\rightarrow} A$ ), then the corresponding antifields are related "the other way round" (i.e., $A * \stackrel{\delta_{\tau}}{\rightarrow} c^{*}$ ). This feature represents a useful guideline for dealing with more complex models or field contents. Using Eq. (38) and $s \widetilde{A}=-F^{\tilde{A}}$, it can be explicitly shown that $\left[s, \delta_{\tau}\right] \widetilde{A}=\mathcal{L}_{\tau} \widetilde{A}$, i.e., the VSUSY algebra is satisfied off-shell for all fields of the geometric sector. (In fact, this result holds by construction [16].)

In the sequel, we will sketch the general procedure for obtaining the VSUSY variations of all fields and antifields on $\mathbf{R}^{d}$. A $p$-form gauge potential $X_{p}$ generally admits a hierarchy of ghosts $X_{p-1}^{1}, X_{p-2}^{2}, \ldots$ and the gauge-fixing of the corresponding symmetries leads to analogous hierarchies of 
TABLE I. $X_{p}$ pyramid.

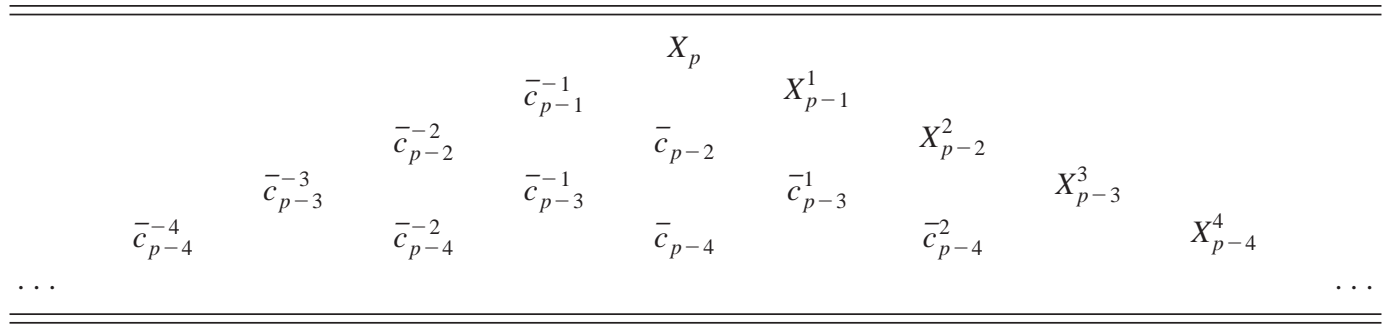

antighosts. All of these fields can be organized in a $B V$ pyramid culminating in $X_{p}$, see Table I.

For a complete ladder $\widetilde{\Omega}$, the VSUSY variations are postulated to be given by

$$
\delta_{\tau} \widetilde{\Omega}=i_{\tau} \widetilde{\Omega}
$$

i.e., VSUSY climbs the ladder from the highest ghost number to the lowest one. The variations of the classical fields, the ghosts, and the associated antifields follow directly from Eq. (40) by choosing $\widetilde{\Omega}=\widetilde{X}_{p}, \widetilde{Y}_{d-p-1}$. As noted after Eq. (39), the antifields transform in the other direction than the fields do.

Next, we consider the antighosts with negative ghost number, i.e., those located on the left half of the BV pyramid, i.e., $\bar{c}_{p-1}^{-1}, \bar{c}_{p-2}^{-2}, \bar{c}_{p-3}^{-1}, \ldots$. Their variations follow from the arguments preceding Eqs. (A30) of the Appendix. Those of the associated antifields are inferred from the general guideline that antifields transform in the other direction than fields do, i.e., $\bar{C}^{\alpha} \stackrel{\delta_{\tau}}{\rightarrow} \bar{C}^{\beta}$ implies $\bar{C}_{\beta}^{*} \stackrel{\delta_{\tau}}{\rightarrow} \bar{C}_{\alpha}^{*}$, cf., Eq. (A31) below.

All of the remaining antighosts have a positive (more precisely non-negative) ghost number. Those which have the same total degree can be gathered in ladders which correspond to the diagonals on the right half of the pyramid:

$$
\begin{aligned}
& \widetilde{\bar{c}}_{p-2}=\bar{c}_{p-2}+\bar{c}_{p-3}^{1}+\cdots+\bar{c}^{p-2}, \\
& \widetilde{\bar{c}}_{p-4}=\bar{c}_{p-4}+\bar{c}_{p-5}^{1}+\cdots+\bar{c}^{p-4}, \ldots .
\end{aligned}
$$

These generalized forms are incomplete since they only involve components with positive ghost number. ${ }^{1}$ Similarly, the antifields associated to the antighosts (41) can be collected into ladders containing only components with negative ghost number:

$$
\left(\widetilde{\bar{c}}_{p-2}\right)^{*}=\left(\bar{c}^{p-2}\right) *+\cdots+\left(\bar{c}_{p-2}\right) *, \ldots
$$

The transformation law (40) is now postulated for all of these ladders, i.e., for $\widetilde{\Omega}=\widetilde{\bar{c}}_{p-2 n},\left(\widetilde{\bar{c}}_{p-2 n}\right)^{*}$ with $n$ $=1,2, \ldots$.

\footnotetext{
${ }^{1}$ We note that the BV pyramid does not involve the complete ladder $\widetilde{X}_{p}$ either, but only those components which have positive ghost number.
}

The $\delta_{\tau}$-variations of the multipliers $\Pi^{\alpha}=s \bar{C}^{\alpha}$ follow from the variations of the $\bar{C}^{\alpha}$ by requiring the VSUSY algebra $\left[s, \delta_{\tau}\right]=\mathcal{L}_{\tau}$ to be satisfied:

$$
\delta_{\tau} \Pi^{\alpha}=\delta_{\tau} s \bar{C}^{\alpha}=\mathcal{L}_{\tau} \bar{C}^{\alpha}-s\left(\delta_{\tau} \bar{C}^{\alpha}\right) .
$$

The antifields $\left(\bar{C}_{\alpha}^{*}, \Pi_{\alpha}^{*}\right)$ associated with the BRST doublets $\left(\bar{C}^{\alpha}, \Pi^{\alpha}\right)$ again transform "the other way round."

For instance, for Chern-Simons theory, the $\delta_{\tau}$ variation of the antighost $\bar{c}$ has to vanish for dimensional and ghostnumber reasons ("there is nothing it can transform into") and the argument (43) then yields the $\delta_{\tau}$ variation of the auxiliary field $b \equiv s \bar{c}$ :

$$
\delta_{\tau} \bar{c}=0 \Rightarrow \delta_{\tau} b=\delta_{\tau} s \bar{c}=\left(\mathcal{L}_{\tau}-s \delta_{\tau}\right) \bar{c}=\mathcal{L}_{\tau} \bar{c}
$$

The associated doublet of antifields $\left(\bar{c}^{*}, b^{*}\right)$ is assumed to transform "the other way round:"

$$
\delta_{\tau} b^{*}=0, \quad \delta_{\tau} \bar{c}^{*}=\mathcal{L}_{\tau} b^{*}
$$

\section{B. Ward identity}

Consider again Chern-Simons theory for concreteness. The transformation law (38) induces the following variation of the minimal BV action (25):

$$
\begin{aligned}
\delta_{\tau} S_{\min } & =\left.\frac{1}{2} \int_{\mathbf{R}^{3}} \operatorname{tr}\left\{\tilde{A} \mathcal{L}_{\tau} \widetilde{A}\right\}\right|_{3} ^{-1} \\
& =\int_{\mathbf{R}^{3}} \operatorname{tr}\left\{A^{*} \mathcal{L}_{\tau} A+c^{*} \mathcal{L}_{\tau} c\right\} .
\end{aligned}
$$

The transformations (44) and (45) yield a similar expression for the variation of the auxiliary action:

$$
\delta_{\tau} S_{\text {aux }}=-\delta_{\tau} \int_{\mathbf{R}^{3}} \operatorname{tr}\left\{\bar{c}^{*} b\right\}=\int_{\mathbf{R}^{3}} \operatorname{tr}\left\{b^{*} \mathcal{L}_{\tau} b+\bar{c}^{*} \mathcal{L}_{\tau} \bar{c}\right\}
$$

Thus, the nonminimal BV action $S_{\mathrm{nm}}=S_{\min }+S_{\mathrm{aux}}$ satisfies a broken Ward identity:

$$
\begin{aligned}
\mathcal{W}_{\tau} S_{\mathrm{nm}} & \equiv \int_{\mathbf{R}^{3}} \operatorname{tr}\left\{\delta_{\tau} \Phi^{A} \frac{\delta S_{\mathrm{nm}}}{\delta \Phi^{A}}+\delta_{\tau} \Phi_{A}^{*} \frac{\delta S_{\mathrm{nm}}}{\delta \Phi_{A}^{*}}\right\} \\
& =\int_{\mathbf{R}^{3}} \operatorname{tr}\left\{\Phi_{A}^{*} \mathcal{L}{ }_{\tau} \Phi^{A}\right\} .
\end{aligned}
$$


The breaking is linear in the fields (and also in the antifields). Henceforth, it is unproblematic for the quantum theory since "insertions" that are linear in quantum fields are not renormalized by quantum corrections. We note that the result (46) can also be derived from the expression $S_{\mathrm{nm}}=S_{\mathrm{inv}}$ $-\int_{\mathbf{R}^{3}} \operatorname{tr}\left\{\Phi_{A}^{*} s \Phi^{A}\right\}$ by substituting the variations (39), (44), and (45), and using $\left[s, \delta_{\tau}\right]=\mathcal{L}_{\tau}$.

After elimination of the antifields, the Ward identity (46) takes the form

$$
\begin{aligned}
\mathcal{W}_{\tau} \Sigma & \equiv \int_{\mathbf{R}^{3}} \operatorname{tr}\left\{\delta_{\tau} \Phi^{A} \frac{\delta \Sigma}{\delta \Phi^{A}}+\delta_{\tau} \rho_{A} \frac{\delta \Sigma}{\delta \rho_{A}}\right\} \\
& =-\int_{\mathbf{R}^{3}} \operatorname{tr}\left\{\rho_{A} \mathcal{L}_{\tau} \Phi^{A}\right\} .
\end{aligned}
$$

It has the same form as the one found in the BRST framework $[8,31]$ where no external sources are introduced for the fields $\bar{c}$ and $b$. Yet, it is their inclusion which leads to the quite symmetric expression (47).

The fact that the breaking is linear in the quantum fields and in the sources only holds in the Landau gauge $d \star A$ $=0$ : in a different gauge, $d \star A-\alpha / 2 \star b=0$ with $\alpha \in \mathbf{R}^{*}$, the breaking term is nonlinear in the quantum fields.

To summarize the two previous sections, we can say that the BV approach readily leads to a VSUSY algebra which closes off shell and to a Ward identity which is broken by a term that is linear in the quantum fields and sources (in the Landau gauge).

\section{On the algebra of symmetries}

It is interesting to compare the action of the operators $\delta_{\tau}$ and $s$ on the basic fields (considering again Chern-Simons theory for illustration). For this purpose, we decompose $s$ according to $s=s_{0}+s_{1}$ where $s_{0}$ and $s_{1}$ represent, respectively, the linear and nonlinear parts of the operator. By virtue of $s \widetilde{A}=-F^{\tilde{A}}$, the action of $s_{0}$ on the generalized field $\widetilde{A}$ is given by

$$
s_{0} \widetilde{A}=-d \widetilde{A} .
$$

Comparison with Eq. (38) shows that each of the operators $s_{0}$ and $\delta_{\tau}$ acts in the same fashion on all fields occurring in the expansion $\widetilde{A}$. However, the two operators act in opposite directions inside the ladder $\widetilde{A}$ : while $s_{0}$ increases the ghost number by one unit, $\delta_{\tau}$ lowers it by the same amount,

$$
\begin{aligned}
& s_{0} \\
& \rightarrow \\
& \widetilde{A}=c^{*}+A^{*}+A+c, \\
& \leftarrow
\end{aligned}
$$

both operators being related by

$$
\left[s_{0}, \delta_{\tau}\right]=\mathcal{L}_{\tau} .
$$

The linear part $s_{0}$ of the $s$ operator (which already determines the non-Abelian structure of the theory to a large extent [37]) also allows for a unified formulation of all symmetries. To present this geometric description, we define (in analogy to $\widetilde{d}=d+s$ )

$$
\begin{aligned}
& \widetilde{d}_{0} \equiv d+s_{0}, \\
& \tilde{i}_{\tau} \equiv i_{\tau}-\delta_{\tau} .
\end{aligned}
$$

The horizontality condition (48) defining $s_{0}$ and the symmetry condition (38) defining $\delta_{\tau}$ are then equivalent to

$$
\widetilde{d}_{0} \tilde{A}=0, \quad \tilde{i}_{\tau} \tilde{A}=0
$$

and the compatibility condition for these two equations,

$$
0=\left[\widetilde{d}_{0}, \tilde{i}_{\tau}\right]=\left[d, i_{\tau}\right]-\left[s_{0}, \delta_{\tau}\right]
$$

is the VSUSY-algebra relation (50).

\section{CONCLUSION}

As is well-known, the BV formalism represents a systematic procedure for constructing an $s$-invariant action in the case of a gauge algebra which is reducible and/or only valid on shell. The $s$ operator of the BV-setting is nothing but the linearized Slavnov-Taylor operator. If the symmetry algebra is only valid on shell, antifields appear in the $s$ variations and the solution $\left.S_{\mathrm{nm}}\right|_{\Phi_{A}^{*}}$ of the Slavnov-Taylor identity involves terms that are quadratic (or of higher order) in the antifields.

The algebraic framework for the BV formalism on which we elaborated here represents an elegant procedure for constructing solutions of the Slavnov-Taylor identity for topological models of Schwarz-type as defined in various dimensions. In particular, it allows us to obtain quite straightforwardly the VSUSY transformations which are most useful for dealing with the quantum version of these theories. As pointed out above, deeper geometric insights into the BV framework should explain more fully why highly nontrivial solutions of the master equation can be obtained from such a simple algebraic procedure.

As emphasized in Sec. III, topological models of Wittentype can also be introduced along these lines. However, their BRST and VSUSY algebras close off shell in the standard BRST approach and therefore the introduction of antifields is not useful for their description [16,24,27].

Our discussion of VSUSY for topological models defined on flat space-time can be generalized to generic manifolds by incorporating VSUSY in the $s$ operation: this leads to an exact rather than a broken Ward identity and it proves to be useful for discussing the relationship between topological models and gravity [18].

\section{ACKNOWLEDGMENTS}

The work of J.M.G. was supported by "The Danish Research Academy." The work of T.P. was supported by the "Fonds zur Förderung der Wissenschaflichen Forschung" under Project Grant Number P11582-PHY. F.G. wishes to 
thank M. Schweda and all the members of the Institut für Theoretische Physik of the Technical University of Vienna for the warm hospitality extended to him during part of the present work. He also acknowledges enlightening discussions with O. Piguet, F. Delduc, and S. Wolf.

\section{APPENDIX: EXAMPLE: $B F$ MODEL IN $\mathrm{R}^{4}$}

In this Appendix, we discuss the symmetries of the 4D $B F$ model using the algebraic approach to the $\mathrm{BV}$ formalism outlined in Sec. III B. We will carry out explicitly the elimination of antifields so as to allow for a comparison with the results obtained from other lines of reasoning (in particular with those obtained from the standard BRST approach).

\section{Symmetries of the classical action}

\section{a. The model and its symmetries}

The $B F$ model in $\mathbf{R}^{4}$ involves two gauge potentials: the YM 1 -form $A$ and the 2-form potential $B \equiv B_{2}^{0}$, i.e., a Lie algebra-valued 2-form transforming under the adjoint representation of the gauge group. The model is characterized by the action

$$
S_{\text {inv }}[A, B]=\int_{\mathbf{R}^{4}} \operatorname{tr}\{B F\},
$$

which leads to the equations of motion

$$
F=0 \quad \text { and } \quad D B=0 .
$$

The functional (A1) is not only invariant under ordinary gauge transformations, but also under the local symmetry

$$
\delta B=D B_{1} .
$$

By virtue of the second Bianchi identity $D\left(D B_{0}\right)=\left[F, B_{0}\right]$ and the equation of motion $F=0$, the right-hand side of Eq. (A3) is on-shell invariant under the transformation $\delta B_{1}$ $=D B_{0}$. Thus, the symmetry (A3) is one-stage reducible on shell.

\section{b. Horizontality conditions and s transformations}

Apart from the ghost $c$ parametrizing ordinary gauge transformations, we have ghosts $B_{1}^{1} \equiv B_{1}$ and $B_{0}^{2} \equiv B_{0}$ parametrizing the reducible symmetry (A3). Thus, one introduces generalized forms $[1]$

$$
\begin{aligned}
\widetilde{A} & =A_{4}^{-3}+A_{3}^{-2}+A_{2}^{-1}+A+c \\
& =B_{0}^{*}+B_{1}^{*}+B^{*}+A+c, \\
\widetilde{B} & =B_{4}^{-2}+B_{3}^{-1}+B+B_{1}^{1}+B_{0}^{2} \\
& =c^{*}+A^{*}+B+B_{1}+B_{0},
\end{aligned}
$$

where $B_{0}^{*} \equiv\left(B_{0}\right)^{*}, B_{1}^{*} \equiv\left(B_{1}\right)^{*}$ and where the identification of antifields has simply been performed by considering the index structure of all fields (see Sec. II). The gauge potentials
$A$ and $B$ and, more generally, the generalized fields $\widetilde{A}$ and $\widetilde{B}$ can be viewed as dual to each other (see Sec. III A).

In view of the equations of motion (A2), one postulates the horizontality conditions [1]

$$
\widetilde{F}=0 \quad \text { and } \quad \widetilde{D} \widetilde{B}=0,
$$

where $\widetilde{F}$ and $\widetilde{D}$ are defined by Eq. (20). The previous relations are equivalent to

$$
s \widetilde{A}=-F^{\widetilde{A}} \quad \text { and } \quad s \widetilde{B}=-D^{\widetilde{A}} \widetilde{B}
$$

and thereby determine all $s$ variations: by substitution of expressions (A4), one obtains

$$
\begin{aligned}
s c=-\frac{1}{2}[c, c], \quad s B^{*} & =-F-\left[c, B^{*}\right] \\
s A=-D c, \quad s B_{1}^{*} & =-D B^{*}-\left[c, B_{1}^{*}\right] \\
s B_{0}^{*} & =-D B_{1}^{*}-\left[c, B_{0}^{*}\right]-\frac{1}{2}\left[B^{*}, B^{*}\right]
\end{aligned}
$$

and

$$
\begin{aligned}
& s B_{0}=-\left[c, B_{0}\right], \\
& s B_{1}=-D B_{0}-\left[c, B_{1}\right], \\
& s B=-D B_{1}-[c, B]-\left[B^{*}, B_{0}\right], \\
& s A^{*}=-D B-\left[c, A^{*}\right]-\left[B^{*}, B_{1}\right]-\left[B_{1}^{*}, B_{0}\right], \\
& s c^{*}=-D A^{*}-\left[c, c^{*}\right]-\left[B^{*}, B\right]-\left[B_{1}^{*}, B_{1}\right]-\left[B_{0}^{*}, B_{0}\right] .
\end{aligned}
$$

The fields and antifields of the minimal sector can be collected in $\left(\Phi^{a}\right)=\left(A, c, B, B_{1}, B_{0}\right)$ and $\left(\Phi_{a}^{*}\right)$ $=\left(A^{*}, c^{*}, B^{*}, B_{1}^{*}, B_{0}^{*}\right)$. By construction, the $s$ variations of these variables as given by Eqs. (A7) and (A8) are nilpotent off shell. The fact that the transformation law of the classical field $B$ involves the antifield $B^{*}$ reflects the fact that the symmetry algebra generated by Eq. (A3) closes only on shell. If all antifields are set to zero, one recovers the standard BRST transformations of $\left(A, c, B, B_{1}, B_{0}\right)$ which are only nilpotent on the mass-shell.

\section{c. Minimal BV action}

Proceeding along the lines of Sec. III B, we can extend the classical action (A1). From the horizontality conditions (A6), we obtain the cocycle condition [12]

$$
s \operatorname{tr}\left\{\widetilde{B} F^{\tilde{A}}\right\}=-d \operatorname{tr}\left\{\widetilde{B} F^{\tilde{A}}\right\},
$$

which yields the $s$-invariant minimal $B V$ action $[1,12]$

$$
\left.S_{\min }\left[\Phi^{a} ; \Phi_{a}^{*}\right] \equiv \int_{\mathbf{R}^{4}} \operatorname{tr}\left\{\widetilde{B} F^{\widetilde{A}}\right\}\right|_{4} ^{0} .
$$

Substitution of the expansions (A4) leads to the explicit expression 


$$
\begin{aligned}
S_{\text {min }}= & S_{\text {inv }}-\int_{\mathbf{R}^{4}} \operatorname{tr}\left\{\Phi_{a}^{*} s \Phi^{a}\right\} \\
& -\frac{1}{2} \int_{\mathbf{R}^{4}} \operatorname{tr}\left\{B^{*}\left[B^{*}, B_{0}\right]\right\},
\end{aligned}
$$

where the last term reflects the antifield dependence of the transformation law of $B$. We note that all of the $s$ variations (A7) and (A8) have the BV form (3) which confirms the identification of antifields made in Eq. (A4).

\section{d. Gauge fixing and elimination of antifields}

Gauge fermion and auxiliary fields. Gauge fixing of all symmetries, i.e., of YM invariance and of the reducible symmetry of the 2 -form potential $B$, requires a gauge fermion of the form

$$
\begin{aligned}
\Psi_{\mathrm{gf}}= & \int_{\mathbf{R}^{4}} \operatorname{tr}\left\{\bar{c} d \star A+\bar{c}_{1}^{-1} d \star B+\bar{c}^{-2} d \star B_{1}\right. \\
& \left.+\bar{c}^{0}\left(d \star \bar{c}_{1}^{-1}+\alpha \star \pi^{-1}\right)\right\} \quad(\alpha \in \mathbf{R}) .
\end{aligned}
$$

The involved antighosts $\left(\bar{C}^{\alpha}\right) \equiv\left(\bar{c}, \bar{c}_{1}^{-1}, \bar{c}^{-2}, \bar{c}^{0}\right)$ are supplemented with auxiliary fields $\left(\Pi^{\alpha}\right) \equiv\left(b, \pi_{1}, \pi^{-1}, \pi^{1}\right)$ so as to define BRST doublets:

$$
\begin{aligned}
& s \bar{c}=b, \quad s \bar{c}_{1}^{-1}=\pi_{1}, \quad s \bar{c}^{-2}=\pi^{-1}, \quad s \bar{c}^{0}=\pi^{1}, \\
& s b=0, \quad s \pi_{1}=0, \quad s \pi^{-1}=0, \quad s \pi^{1}=0 .
\end{aligned}
$$

The corresponding antifields transform in a dual way [cf. Eq. (7)],

$$
\begin{aligned}
s \bar{c}^{*} & =0, \quad s\left(\bar{c}_{1}^{-1}\right)^{*}=0, \quad s\left(\bar{c}^{-2}\right)^{*}=0, \quad s\left(\bar{c}^{0}\right)^{*}=0, \\
s b^{*} & =\bar{c}^{*}, \quad s\left(\pi_{1}\right)^{*}=-\left(\bar{c}_{1}^{-1}\right)^{*}, \\
s\left(\pi^{-1}\right)^{*} & =-\left(\bar{c}^{-2}\right)^{*}, \quad s\left(\pi^{1}\right)^{*}=-\left(\bar{c}^{0}\right)^{*}
\end{aligned}
$$

and thereby ensure the $s$ invariance of the auxiliary functional $S_{\text {aux }}=-\int_{\mathbf{R}^{4}} \operatorname{tr}\left\{\left(\bar{C}^{\alpha}\right)^{*} \Pi_{\alpha}\right\}$ which gives rise to the nonminimal action $S_{\mathrm{nm}}=S_{\min }+S_{\mathrm{aux}}$.

Elimination of antifields. Since antifields have been associated to all fields, external sources are also introduced for each field (and not only for those transforming nonlinearly under the $s$ operation, as is usually done in the BRST approach). Altogether, we have the fields $\left(\Phi^{A}\right)=\left(\Phi^{a}, \bar{C}^{\alpha}, \Pi^{\alpha}\right)$ with

$$
\begin{aligned}
& \left(\Phi^{a}\right)=\left(A, c, B, B_{1}, B_{0}\right), \quad\left(\bar{C}^{\alpha}\right)=\left(\bar{c}, \bar{c}_{1}^{-1}, \bar{c}^{-2}, \bar{c}^{0}\right), \\
& \left(\Pi^{\alpha}\right)=\left(b, \pi_{1}, \pi^{-1}, \pi^{1}\right)
\end{aligned}
$$

and the associated external sources $\left(\rho_{A}\right)$ are to be denoted as follows:

$$
\begin{aligned}
& \left(\gamma, \sigma, \rho_{2}^{-1}, \rho_{3}^{-2}, \rho_{4}^{-3}\right), \quad\left(\bar{\sigma}, \bar{\sigma}_{3}^{0}, \bar{\sigma}_{4}^{1}, \bar{\sigma}_{4}^{-1}\right), \\
& \left(\lambda, \lambda_{3}^{-1}, \lambda_{4}^{0}, \lambda_{4}^{-2}\right) .
\end{aligned}
$$

The antifields $\left(\Phi_{A}^{*}\right)$ will now be expressed in terms of these sources by virtue of the prescription (10). For the antifields of the minimal sector, this entails

$$
\begin{aligned}
& A^{*}=-(\gamma-\star d \bar{c}) \equiv-\hat{\gamma}, \quad c^{*}=-\sigma, \\
& B^{*}=-\left(\rho_{2}^{-1}+\star d \bar{c}_{1}^{-1}\right) \equiv-\hat{\rho}_{2}^{-1}, \\
& B_{1}^{*}=-\left(\rho_{3}^{-2}-\star d \bar{c}^{-2}\right) \equiv-\hat{\rho}_{3}^{-2}, \\
& B_{0}^{*}=-\rho_{4}^{-3}
\end{aligned}
$$

whereas the antifields associated to the antighosts and multiplier fields are given by

$$
\begin{aligned}
\bar{c}^{*} & =-(\bar{\sigma}+d \star A) \equiv-\hat{\bar{\sigma}} \\
\left(\bar{c}_{1}^{-1}\right) * & =-\left(\bar{\sigma}_{3}^{0}-d \star B-\star d \bar{c}^{0}\right) \equiv-\hat{\bar{\sigma}}_{3}^{0}, \\
\left(\bar{c}^{-2}\right) * & =-\left(\bar{\sigma}_{4}^{1}-d \star B_{1}\right) \equiv-\hat{\bar{\sigma}}_{4}^{1}, \\
\left(\bar{c}^{0}\right)^{*} & =-\left(\bar{\sigma}_{4}^{-1}-\alpha \star \pi^{-1}-d \star \bar{c}_{1}^{-1}\right) \equiv-\hat{\bar{\sigma}}_{4}^{-1}, \\
b^{*} & =-\lambda, \\
\left(\pi_{1}\right)^{*} & =-\lambda_{3}^{-1}, \\
\left(\pi^{-1}\right)^{*} & =-\left(\lambda_{4}^{0}+\alpha \star \bar{c}^{0}\right) \equiv-\hat{\lambda}_{4}^{0}, \\
\left(\pi^{1}\right)^{*} & =-\lambda_{4}^{-2} .
\end{aligned}
$$

Vertex functional. The gauge-fixed action including external sources is obtained from $S_{\mathrm{nm}}=S_{\min }+S_{\text {aux }}$ by eliminating antifields according to relations (A16) and (A17). This leads to

$$
\begin{aligned}
\Sigma & =S_{\text {inv }}+\int_{\mathbf{R}^{4}} \operatorname{tr}\left\{\hat{\rho}_{A} s \Phi^{A}\right\}+S_{\text {quadr }} \\
& =S_{\text {inv }}+s \Psi_{\text {gf }}+\int_{\mathbf{R}^{4}} \operatorname{tr}\left\{\rho_{A} s \Phi^{A}\right\}+S_{\text {quadr }} \\
& =S_{\text {inv }}+S_{\text {gf }}+S_{\text {ext }}+S_{\text {quadr }},
\end{aligned}
$$

where

$$
S_{\text {quadr }}=-\frac{1}{2} \int_{\mathbf{R}^{4}} \operatorname{tr}\left\{B_{0}\left[\hat{\rho}_{2}^{-1}, \hat{\rho}_{2}^{-1}\right]\right\}
$$

is related to the fact that the $s$ variation of $B$ exhibits an antifield dependence, see Eq. (A8). For $\rho_{A}=0$, expression (A18) coincides with the one of Ref. [7] in which external sources are introduced at a different stage.

$s$ variations. After elimination of all antifields in terms of sources, the $s$ variations of the basic fields $\left(A, c, B, B_{1}, B_{0}\right)$ are exactly the same as before except for the fact that $s B$ now depends on a (hatted) source rather than an antifield:

$$
s B=-D B_{1}-[c, B]+\left[\hat{\rho}_{2}^{-1}, B_{0}\right] .
$$


The sources associated to the basic fields transform as

$$
\begin{aligned}
s \gamma & =D B-\left[\hat{\rho}_{3}^{-2}, B_{0}\right]-\left[\hat{\rho}_{2}^{-1}, B_{1}\right]-[c, \hat{\gamma}]-\star d b, \\
s \sigma & =-D \hat{\gamma}-\left[\rho_{4}^{-3}, B_{0}\right]-\left[\hat{\rho}_{3}^{-2}, B_{1}\right]-\left[\hat{\rho}_{2}^{-1}, B\right]-[c, \sigma], \\
s \rho_{2}^{-1} & =F-\left[c, \hat{\rho}_{2}^{-1}\right]+\star d \pi_{1}, \\
s \rho_{3}^{-2} & =-D \hat{\rho}_{2}^{-1}-\left[c, \hat{\rho}_{3}^{-2}\right]-\star d \pi^{-1}, \\
s \rho_{4}^{-3} & =-D \hat{\rho}_{3}^{-2}-\left[c, \rho_{4}^{-3}\right]+\frac{1}{2}\left[\hat{\rho}_{2}^{-1}, \hat{\rho}_{2}^{-1}\right],
\end{aligned}
$$

and those associated to the antighosts and multipliers transform as

$$
\begin{aligned}
s \bar{\sigma} & =-d \star D c, \\
s \bar{\sigma}_{3}^{0} & =d \star\left(D B_{1}+[c, B]-\left[\hat{\rho}_{2}^{-1}, B_{0}\right]\right)-\star d \pi^{1}, \\
s \bar{\sigma}_{4}^{1} & =d \star\left(D B_{0}+\left[c, B_{1}^{1}\right]\right), \\
s \bar{\sigma}_{4}^{-1} & =-d \star \pi_{1}, \\
s \lambda & =\bar{\sigma}+d \star A, \\
s \lambda_{3}^{-1} & =-\bar{\sigma}_{3}^{0}+d \star B+\star d \bar{c}^{0}, \\
s \lambda_{4}^{0} & =-\bar{\sigma}_{4}^{1}+d \star B_{1}^{1}-\alpha \star \pi^{1}, \\
s \lambda_{4}^{-2} & =-\bar{\sigma}_{4}^{-1}+\alpha \star \pi^{-1}+d \star \bar{c}_{1}^{-1} .
\end{aligned}
$$

The $s$ variations of fields and sources all have the form

$$
{ }_{s} \Phi^{A}=\frac{\delta \Sigma}{\delta \rho_{A}}, \quad s \rho_{A}=\frac{\delta \Sigma}{\delta \Phi^{A}}
$$

and can be viewed as the relict of the BV variations (14) after the elimination of all antifields. Obviously, the $s$ variations (A23) determine the linearized Slavnov-Taylor operator, i.e.,

$$
s=\mathcal{S}_{\Sigma} \equiv \int \operatorname{tr}\left\{\frac{\delta \Sigma}{\delta \Phi^{A}} \frac{\delta}{\delta \rho_{A}}+\frac{\delta \Sigma}{\delta \rho_{A}} \frac{\delta}{\delta \Phi^{A}}\right\}
$$

Thus, the BV master equation (13) (with antifields expressed in terms of sources) represents the Slavnov-Taylor identity

$$
\mathcal{S}(\Sigma) \equiv \int \operatorname{tr}\left\{\frac{\delta \Sigma}{\delta \Phi^{A}} \frac{\delta \Sigma}{\delta \rho_{A}}\right\}=0
$$

which ensures that $s^{2}=0$ off shell and that $s \Sigma=\mathcal{S}_{\Sigma}(\Sigma)$ $=2 \mathcal{S}(\Sigma)=0$. This interpretation of the master equation is a cornerstone of the theory and is further discussed in the literature, both at the classical and quantum level $[9,11]$. Here, we only note that the hatted sources associated to antighosts and multipliers form BRST doublets [see Eqs. (A13) and (A17)] which simplifies the cohomogical analysis of the quantum theory.

\section{e. $B V$ versus BRST}

Let us summarize the conclusions that we can draw from our discussion of the BF model at the classical level (and which are in accordance with the general results $[9,11])$. For an off-shell, irreducible symmetry (such as YM invariance), the differences between the BV approach and the BRST procedure are twofold.

The $s$ operator of the BV formalism represents the linearized Slavnov-Taylor operator: unlike the standard BRST differential, this operator does not leave the external sources invariant. [The nontrivial transformation laws of the sources follow from the nontrivial $s$ variations of antifields given by Eqs. (14), after the elimination of antifields in terms of sources by virtue of the gauge fermion $\Psi_{\text {gf }}$.]

In the BV approach, one introduces sources for all fields, not only for those transforming nonlinearly, as one usually does in the BRST framework. Of course, the latter framework also allows for the inclusion of such sources: although they are not particularly useful, they lead to a more symmetric expression for the Slavnov-Taylor identity [and also for the Ward identities, see Eq. (A33) below and comments thereafter].

For the local symmetry of the 2 -form potential $B$ (which represents a reducible symmetry), an extra feature appears in the BV formulation.

The $s$ variation of the classical field $B$ depends on sources (thereby ensuring the off-shell nilpotency of the $s$ operator). Another facet of this issue is the presence of the functional $S_{\text {quadr }}$ in the vertex functional. While the BV approach automatically produces such contributions which are nonlinear in the external sources, they have to be added "by hand" in the standard BRST framework, ${ }^{2}$ e.g., see $[5,7,38]$.

\section{VSUSY}

\section{a. VSUSY transformations of fields}

As in the standard BRST approach [16], we start from the $\varnothing$-symmetry conditions

$$
\delta_{\tau} \widetilde{A}=i_{\tau} \widetilde{A}, \quad \delta_{\tau} \widetilde{B}=i_{\tau} \widetilde{B} .
$$

These relations are then spelled out in terms of component fields [as in Eq. (39)] and the antifields are eliminated in terms of sources. In this way, we obtain the VSUSY variations of the basic fields,

\footnotetext{
${ }^{2}$ It should be noted that an off-shell formulation for the basic $s$ variations can eventually be given within the BRST framework by mimicking the BV approach, see Ref. [8].
} 


$$
\begin{aligned}
& \delta_{\tau} c=i_{\tau} A, \quad \delta_{\tau} B_{0}=i_{\tau} B_{1}, \\
& \delta_{\tau} A=-i_{\tau} \hat{\rho}_{2}^{-1}, \quad \delta_{\tau} B_{1}=i_{\tau} B \\
& \delta_{\tau} B=-i_{\tau} \hat{\gamma}
\end{aligned}
$$

and the variations of the associated sources:

$$
\begin{aligned}
\delta_{\tau} \hat{\gamma} & =i_{\tau} \sigma, \quad \delta_{\tau} \sigma=0, \\
\delta_{\tau} \hat{\rho}_{2}^{-1} & =i_{\tau} \hat{\rho}_{3}^{-2}, \quad \delta_{\tau} \hat{\rho}_{3}^{-2}=i_{\tau} \rho_{4}^{-3}, \\
\delta_{\tau} \rho_{4}^{-3} & =0 .
\end{aligned}
$$

We now define the VSUSY variations of the antighosts $\left(\bar{C}^{\alpha}\right)=\left(\bar{c}, \bar{c}_{1}^{-1}, \bar{c}^{-2}, \bar{c}^{0}\right)$ in such a way that relations (A28) hold for the unhatted sources, i.e., such that we have

$$
\begin{aligned}
\delta_{\tau} \gamma & =i_{\tau} \sigma, \quad \delta_{\tau} \sigma=0, \\
\delta_{\tau} \rho_{2}^{-1} & =i_{\tau} \rho_{3}^{-2}, \quad \delta_{\tau} \rho_{3}^{-2}=i_{\tau} \rho_{4}^{-3}, \\
\delta_{\tau} \rho_{4}^{-3} & =0 .
\end{aligned}
$$

The transformation laws of the multipliers $\left(\Pi^{\alpha}\right)$ are then determined by the requirement that the VSUSY algebra is satisfied, see Eq. (44). Altogether, we find the following variations of antighosts and multipliers:

$$
\begin{aligned}
\delta_{\tau} \bar{c} & =0, \quad \delta_{\tau} b=\mathcal{L}_{\tau} \bar{c} \\
\delta_{\tau} \bar{c}_{1}^{-1} & =g(\tau) \bar{c}^{-2}, \quad \delta_{\tau} \pi_{1}=\mathcal{L}_{\tau} \bar{c}_{1}^{-1}+g(\tau) \pi^{-1}, \\
\delta_{\tau} \bar{c}^{-2} & =0, \quad \delta_{\tau} \pi^{-1}=\mathcal{L}_{\tau} \bar{c}^{-2}, \\
\delta_{\tau} \bar{c}^{0} & =0, \quad \delta_{\tau} \pi^{1}=\mathcal{L}_{\tau} \bar{c}^{0} .
\end{aligned}
$$

Here, $g(\tau)=\tau^{\mu} g_{\mu \nu} d x^{\nu}$ denotes the 1-form associated to the vector field $\tau$ by virtue of a space-time metric $\left(g_{\mu \nu}\right)[8,16]$.

If we set all sources to zero, we recover the transformation laws and on-shell VSUSY algebra of the standard BRST approach [16]. If sources are included in the latter framework for the discussion of Ward identities, considerations different from ours lead to the introduction of the hatted sources (A16) and to the variations (A27)-(A30) [7].

To conclude, we come to the $\delta_{\tau}$-variations of the sources (A15) associated to the doublet fields $\left(\bar{C}^{\alpha}, \Pi^{\alpha}\right)$. According to the general guideline indicated after Eq. (39), these sources (antifields) are assumed to transform "the other way round," in the opposite direction as the fields, see Eq. (45):

$$
\begin{gathered}
\delta_{\tau} \bar{\sigma}=\mathcal{L}_{\tau} \lambda-d \star i_{\tau} \hat{\rho}_{2}^{-1}, \\
\delta_{\tau} \lambda=0, \\
\delta_{\tau} \bar{\sigma}_{3}^{0}=-\mathcal{L}_{\tau} \lambda_{3}^{-1}+d \star i_{\tau} \hat{\gamma},
\end{gathered}
$$$$
\delta_{\tau} \lambda_{3}^{-1}=0,
$$$$
\delta_{\tau} \bar{\sigma}_{4}^{1}=-\mathcal{L}_{\tau} \lambda_{4}^{0}-g(\tau) \bar{\sigma}_{3}^{0}+(1-\alpha) \star \mathcal{L}_{\tau} \bar{c}^{0},
$$$$
\delta_{\tau} \lambda_{4}^{0}=-g(\tau) \lambda_{3}^{-1},
$$$$
\delta_{\tau} \bar{\sigma}_{4}^{-1}=-\mathcal{L}_{\tau} \lambda_{4}^{-2}-d \star g(\tau) \bar{c}^{-2}+\alpha \star \mathcal{L}_{\tau} \bar{c}^{-2},
$$

$$
\delta_{\tau} \lambda_{4}^{-2}=0
$$

By construction, the VSUSY algebra is satisfied off shell for all fields and sources.

Ward identity. The $\delta_{\tau}$ variations of fields and antifields induce the broken Ward identity

$$
\mathcal{W}_{\tau} S_{\mathrm{nm}}=\int_{\mathbf{R}^{4}} \operatorname{tr}\left\{ \pm \Phi_{A}^{*} \mathcal{L}_{\tau} \Phi^{A}\right\}
$$

which takes the following form after elimination of antifields:

$$
\mathcal{W}_{\tau} \Sigma=\int_{\mathbf{R}^{4}} \operatorname{tr}\left\{(-1)^{\left|\rho_{A}\right|} \rho_{A} \mathcal{L}{ }_{\tau} \Phi^{A}\right\}
$$

Thus, we have a breaking which is linear in the sources and in the quantum fields [7,31]. It is worthwhile to note that this result has been obtained for an arbitrary value of the gauge parameter $\alpha$. This is in contrast to the usual formulation where VSUSY puts some restrictions on the gauge parameter $[7,8,31]$.

\section{b. BV versus BRST}

Quite generally, we can say the following. Once external sources (associated to nonlinear field variations) are introduced in the BRST framework for discussing Ward identities, one recovers the same results for VSUSY transformations as in the BV approach and also the same type of expression for the breaking of VSUSY. Yet, in the $B V$ framework where sources are introduced for all fields under the disguise of antifields, the VSUSY-breaking term has a more symmetric form.

The algebraic derivation of VSUSY variations (for topological models of Schwarz-type) based on the symmetry conditions (A26) is quite simple and straightforward within the $B V$ formalism whereas the same approach within the standard BRST formalism is fairly involved due to the fact that it refers to the equations of motion [16]. 
[1] H. Ikemori, Class. Quantum Grav. 10, 233 (1993); Mod. Phys. Lett. A 7, 3397 (1992).

[2] D. Birmingham, M. Blau, M. Rakowski, and G. Thompson, Phys. Rep. 209, 129 (1991).

[3] G.T. Horowitz, Commun. Math. Phys. 125, 417 (1989); M. Blau and G. Thompson, Ann. Phys. (N.Y.) 205, 130 (1991); A. Karlhede and M. Roček, Phys. Lett. B 224, 58 (1989).

[4] R.C. Myers and V. Periwal, Phys. Lett. B 225, 352 (1989).

[5] D. Birmingham, M. Rakowski, and G. Thompson, Nucl. Phys. B329, 83 (1990); F. Delduc, F. Gieres, and S.P. Sorella, Phys. Lett. B 225, 367 (1989).

[6] F. Delduc, C. Lucchesi, O. Piguet, and S.P. Sorella, Nucl. Phys. B346, 313 (1990).

[7] E. Guadagnini, N. Maggiore, and S.P. Sorella, Phys. Lett. B 255, 65 (1991); N. Maggiore and S.P. Sorella, Int. J. Mod. Phys. A 8, 929 (1993); C. Lucchesi, O. Piguet, and S.P. Sorella, Nucl. Phys. B395, 325 (1993); A. Blasi and N. Maggiore, Class. Quantum Grav. 10, 37 (1993).

[8] O. Piguet and S.P. Sorella, Algebraic Renormalization (Springer-Verlag, Berlin, 1995); A. Boresch, S. Emery, O. Moritsch, M. Schweda, T. Sommer, and H. Zerrouki, Applications of Noncovariant Gauges in the Algebraic Renormalization Procedure (World Scientific, Singapore, 1998).

[9] I.A. Batalin and G.A. Vilkovisky, Phys. Lett. 102B, 27 (1981); Phys. Rev. D 28, 2567 (1983); J. Gomis, J. Paris, and S. Samuel, Phys. Rep. 259, 1 (1995); G. Barnich, F. Brandt, and M. Henneaux, ibid. 338, 439 (2000); G. Barnich, in Proceedings of the Spring School "QFT and Hamiltonian Systems," Calimanesti, Romania, 2000, hep-th/0011120.

[10] L. Baulieu, M. Bellon, S. Ouvry, and J. Wallet, Phys. Lett. B 252, 387 (1990).

[11] S. Weinberg, The Quantum Theory of Fields (Cambridge University Press, Cambridge, England, 1996), Vol. II.

[12] L. Baulieu, Nucl. Phys. B478, 431 (1996).

[13] L. Baulieu, Phys. Lett. B 441, 250 (1998).

[14] J.C. Wallet, Phys. Lett. B 235, 71 (1990); L. Baulieu, E. Bergshoeff, and E. Sezgin, Nucl. Phys. B307, 348 (1988); M. Abud, J.-P. Ader, and L. Cappiello, Nuovo Cimento A 105, 1507 (1992).

[15] M. Carvalho, L.C. Vilar, C.A. Sasaki, and S.P. Sorella, J. Math. Phys. 37, 5310 (1996).

[16] F. Gieres, J. Grimstrup, T. Pisar, and M. Schweda, J. High Energy Phys. 06, 18 (2000).

[17] T. Pisar, J. Rant, and H. Zerrouki, Mod. Phys. Lett. A 15, 1147 (2000); T. Pisar, Phys. Lett. B 513, 413 (2001).

[18] C.P. Constantinidis, F. Gieres, O. Piguet, and M.S. Sarandy, J. High Energy Phys. 01, 017 (2002).

[19] E. Witten, Nucl. Phys. B311, 46 (1988/89).

[20] O. Piguet, Class. Quantum Grav. 17, 3799 (2000); O. Piguet, talk given at International Conference on Quantization, Gauge
Theory and Strings (Conference dedicated to the memory of Professor Efim Fradkin), Moscow, Russia, 2000, hep-th/0010053.

[21] J. Thierry-Mieg, J. Math. Phys. 21, 2834 (1980); L. Bonora and M. Tonin, Phys. Lett. 98B, 48 (1981); R. Stora, in New Developments in Quantum Field Theory and Statistical Mechanics, Cargèse, 1976, edited by M. Lévy and P. Mitter (NATO ASI Ser. B, Vol. 26) (Plenum, New York, 1977); R. Stora, in Progress in Gauge Field Theory, Cargèse, 1983, edited by G. 't Hooft et al. (NATO ASI Ser. B, Vol. 115) (Plenum, New York, 1984); L. Baulieu and J. Thierry-Mieg, Nucl. Phys. B197, 477 (1982).

[22] R.A. Bertlmann, Anomalies in Quantum Field Theory (Clarendon Press, Oxford, 1996).

[23] L. Baulieu and I.M. Singer, Nucl. Phys. B (Proc. Suppl.) 5B, 12 (1988); S. Ouvry, R. Stora, and P. van Baal, Phys. Lett. B 220, 159 (1989).

[24] A. Brandhuber, O. Moritsch, M.W. de Oliveira, O. Piguet, and M. Schweda, Nucl. Phys. B431, 173 (1994).

[25] L. Baulieu and B. Grossman, Phys. Lett. B 214, 223 (1988).

[26] L. Baulieu and S.L. Shatashvili, J. High Energy Phys. 03, 11 (1999).

[27] F. Gieres, H. Nieder, T. Pisar, L. Popp, and M. Schweda, Mod. Phys. Lett. A 15, 791 (2000).

[28] O.F. Dayi, Mod. Phys. Lett. A 8, 2087 (1993).

[29] L.C. Vilar, S.P. Sorella, and C.A. Sasaki, J. Math. Phys. 40, 2735 (1999).

[30] P. Pasanen, J. Phys. A 29, 8123 (1996).

[31] L.C. Vilar, O.S. Ventura, C.A. Sasaki, and S.P. Sorella, J. Math. Phys. 39, 848 (1998).

[32] L. Baulieu and E. Rabinovici, J. High Energy Phys. 06, 6 (1998).

[33] M. Carvalho, L.C. Vilar, C.A. Sasaki, and S.P. Sorella, J. Math. Phys. 37, 5325 (1996).

[34] A.S. Cattaneo, P. Cotta-Ramusino, F. Fucito, M. Martellini, M. Rinaldi, A. Tanzini, and M. Zeni, Commun. Math. Phys. 197, 571 (1998); A. Accardi, A. Belli, M. Martellini, and M. Zeni, Phys. Lett. B 431, 127 (1998).

[35] A. Schwarz, Commun. Math. Phys. 155, 249 (1993); Lett. Math. Phys. 31, 299 (1994).

[36] N. Maggiore and S.P. Sorella, Nucl. Phys. B377, 236 (1992).

[37] G. Barnich and M. Henneaux, Phys. Lett. B 311, 123 (1993); G. Barnich, M. Henneaux, and R. Tatar, Int. J. Mod. Phys. D 3, 139 (1994); R. Stora (unpublished); A.W. Aste and G. Scharf, Int. J. Mod. Phys. A 14, 3421 (1999).

[38] C. Becchi, Lectures given at the 29th International Universitätswochen für Kernphysik (Schladming, 1990), Genoa University report GEF-TH-10/1990; Lectures given at the ETH Zürich 1996, Genoa University report GEF-TH-96-10, hep-th/9607181. 